\title{
CONCAVE MEROMORPHIC FUNCTIONS INVOLVING CONSTRUCTED OPERATORS
}

\author{
M. Al-Kaseasbeh, M. Darus
}

Abstract. This paper involves constructed differential operators in concave meromorphic function and studied its properties. In particular, coefficient bounds, distortion theorem, and extreme points are obtained.

2010 Mathematics Subject Classification: 33C45.

Keywords: meromorphic function, concave function, constructed operator, distortion theorem, extreme points.

\section{INTRODUCTION}

This paper concerns with class of functions which are analytic in the open unit disk $\mathbb{U}=\{z:|z|<1\}$ except for a simple pole at the origin. Also, this class attains certain geometrical interpretation. Explicitly, mapping $\mathbb{U}$ onto a domain whose complement is unbounded convex set.

Back to analytic functions of the form

$$
f(z)=z+\sum_{k=2}^{\infty} a_{k} z^{k},
$$

it is well-known fact, that the inequality

$$
\Re\left\{1+z \frac{f^{\prime \prime}(z)}{f^{\prime}(z)}\right\}>0, \quad z \in \mathbb{U}
$$

characterises convex functions that map the unit disk onto convex domain.

Due to the similarity, the inequality

$$
\Re\left\{1+z \frac{f^{\prime \prime}(z)}{f^{\prime}(z)}\right\}<0, \quad z \in \mathbb{U}
$$


M. Al-Kaseasbeh, M. Darus - Concave Meromorphic Functions ...

is used sometimes as a definition of concave analytic functions (see e.g [11] and others). However, Bhowmik et al. considered another characterisation of concave analytic functions (see $[6]$ ).

In 2012, the condition (1.2) was used again and shown to be necessary and sufficient condition of concave meromorphic mapping in the form

$$
f(z)=\frac{1}{z}+a_{0}+a_{1} z+\cdots
$$

by Chuaqui, et al. [7].

Further in [7], the coefficients inequality

$$
\left|a_{1}\right|^{2}+3\left|a_{2}\right| \leq 1
$$

was deduced by applied an invariant form of Schwarz's lemma involving with Schwarzian derivative.

Later, Challab and Darus studied on concave meromorphic functions defined by Salagean Operator and Al-Oboudi operator respectively in $[8,9]$.

In [3], the authors estimated $a_{k}$ for $k=2,3, \ldots$ for $f$ of the form

$$
f(z)=\frac{1}{z}+\sum_{k=2}^{\infty} a_{k} z^{k} .
$$

Let us consider the differential operators $R_{\alpha, \lambda}^{n}$ and $D_{\lambda}^{n}$ which introduced respectively in [10] and [4]. Then, the convoluted operator of both of them is

$$
\begin{aligned}
\tilde{D}_{\alpha, \lambda}^{n} f(z) & =D_{\lambda}^{n} f(z) * R_{\alpha, \lambda}^{n} f(z) \\
& =\left(\frac{1}{z}+\sum_{k=2}^{\infty}[1+\lambda(k-1)]^{n} a_{k} z^{k}\right) *\left(\frac{1}{z}+\sum_{k=2}^{\infty}[1+\lambda(k-1)]^{n} C(\alpha, k) a_{k} z^{k}\right) \\
& =\frac{1}{z}+\sum_{k=2}^{\infty}[1+\lambda(k-1)]^{2 n} C(\alpha, k) a_{k}^{2} z^{k}
\end{aligned}
$$

The operator $\tilde{D}_{\alpha, \lambda}^{n}$ introduced in [1].

In the other hand, the authors in [2] introduced new differential operator by means of linear combination of both $R_{\alpha, \lambda}^{n}$ and $D_{\lambda}^{n}$ as follows.

$$
D_{\lambda, \alpha, \gamma}^{n} f(z)=(1-\gamma) R_{\alpha}^{n} f(z)+\gamma D_{\lambda}^{n} f(z), \quad z \in \mathbb{U} .
$$


If $f(z)$ is an meromorphic function of the form $f(z)=\frac{1}{z}+\sum_{k=2}^{\infty} a_{k} z^{k}$, then

$$
D_{\lambda, \alpha, \gamma}^{n} f(z)=\frac{1}{z}+\sum_{k=2}^{\infty}[1+\lambda(k-1)]^{n}[\gamma+(1-\gamma) C(\alpha, k)] a_{k} z^{k}
$$

Now let us define the classes of concave meromorphic functions involving constructed differential operator $D_{\lambda, \alpha, \gamma}^{n}$ as follows.

Definition 1. Let $C_{\Sigma}(\lambda, \alpha, \gamma)$ denote the class of complex functions of the form (1.4) and satisfies

$$
\Re\left\{1+z \frac{\left(D_{\lambda, \alpha, \gamma}^{n} f(z)\right)^{\prime \prime}}{\left(D_{\lambda, \alpha, \gamma}^{n} f(z)\right)^{\prime}}\right\}<0, \quad z \in \mathbb{U}^{*},
$$

where $\lambda, \gamma \geq 0$, and $\alpha, n \in \mathbb{N}_{0}$.

For constructed differential operator $\tilde{D}_{\alpha, \lambda}^{n}$, we define the following class of concave meromorphic functions.

Definition 2. Let $C_{\Sigma}(\lambda, \alpha)$ denote the class of complex functions of the form (1.4) and satisfies

$$
\Re\left\{1+z \frac{\left(\tilde{D}_{\alpha, \lambda}^{n} f(z)\right)^{\prime \prime}}{\left(\tilde{D}_{\alpha, \lambda}^{n} f(z)\right)^{\prime}}\right\}<0, \quad z \in \mathbb{U}^{*},
$$

where $\lambda \geq 0$, and $\alpha, n \in \mathbb{N}_{0}$.

We begin with the coefficient bounds of the classes $C_{\Sigma}(\lambda, \alpha, \gamma)$ and $C_{\Sigma}(\lambda, \alpha)$.

\section{Coefficient Bounds}

First we obtain coefficient bounds of the normalised concave meromorphic functions of the form (1.4) as follow

Theorem 1. Let $f(z)$ be of the form (1.4) and

$$
\sum_{k=2}^{\infty} k^{2}\left|a_{k}\right| \leq 1
$$

Then $f(z)$ is concave meromorphic function. 
Proof. Using the fact that $\Re w \leq 0$ if and only if $\left|\frac{w+1}{w-1}\right|<1$, we need to show that

$$
\left|\frac{1+z \frac{f^{\prime \prime}(z)}{f^{\prime}(z)}+1}{1+z \frac{f^{\prime \prime}(z)}{f^{\prime}(z)}-1}\right|<1
$$

and so

$$
\begin{aligned}
\left|\frac{1+z \frac{f^{\prime \prime}(z)}{f^{\prime}(z)}+1}{1+z \frac{f^{\prime \prime}(z)}{f^{\prime}(z)}-1}\right| & =\left|\frac{2 f^{\prime}(z)+z f^{\prime \prime}(z)}{z f^{\prime \prime}(z)}\right| \\
& =\left|\frac{\frac{-2}{z^{2}}+2 \sum_{k=2}^{\infty} k a_{k} z^{k-1}+\frac{2}{z^{2}}+\sum_{k=2}^{\infty} k(k-1) a_{k} z^{k-1}}{\frac{2}{z^{2}}+\sum_{k=2}^{\infty} k(k-1) a_{k} z^{k-1}}\right| \\
& =\left|\frac{\sum_{k=2}^{\infty}(2 k+k(k-1)) a_{k} z^{k-1}}{\frac{2}{z^{2}}+\sum_{k=2}^{\infty} k(k-1) a_{k} z^{k-1}}\right| \\
& \leq \frac{\sum_{k=2}^{\infty} k(k+1)\left|a_{k}\right|}{2-\sum_{k=2}^{\infty} k(k-1)\left|a_{k}\right|} .
\end{aligned}
$$

The last expression is bounded above by 1 if

$$
\sum_{k=2}^{\infty} k(k+1)\left|a_{k}\right|<2-\sum_{k=2}^{\infty} k(k-1)\left|a_{k}\right|,
$$

which equivalent to (2.1). The other side of the assertion is trivial. Therefore, $f(z)$ is concave meromorphic function.

This result was obtained by the authors in [3]. For classes $C_{\Sigma}(\lambda, \alpha, \gamma)$ and $C_{\Sigma}(\lambda, \alpha)$ we provide the following theorems.

Theorem 2. Let $f(z)$ be of the form (1.4), $\lambda, \gamma \geq 0, \alpha, n \in \mathbb{N}_{0}$ and

$$
\sum_{k=2}^{\infty} k^{2}[1+\lambda(k-1)]^{n}[\gamma+(1-\gamma) C(\alpha, k)]\left|a_{k}\right| \leq 1 .
$$

Then $f(z) \in C_{\Sigma}(\lambda, \alpha, \gamma)$.

Proof. Using the fact that $\Re w \leq 0$ if and only if $\left|\frac{w+1}{w-1}\right|<1$, we need to show that

$$
\left|\frac{1+z \frac{\left(D_{\lambda, \alpha, \gamma}^{n} f\right)^{\prime \prime}(z)}{\left(D_{\lambda, \alpha, \gamma}^{n} f\right)^{\prime}(z)}+1}{1+z \frac{\left(D_{\lambda, \alpha, \gamma}^{n} f\right)^{\prime \prime}(z)}{\left(D_{\lambda, \alpha, \gamma}^{n} f\right)(z)}-1}\right|<1 .
$$

Following the steps of proof Theorem 1, the result is straightforward. 
Theorem 3. Let $f(z)$ be of the form (1.4), $\lambda \geq 0, \alpha, n \in \mathbb{N}_{0}$ and

$$
\sum_{k=2}^{\infty} k^{2}[1+\lambda(k-1)]^{2 n} C(\alpha, k)\left|a_{k}\right|^{2} \leq 1 .
$$

Then $f(z) \in C_{\Sigma}(\lambda, \alpha)$.

Proof. Using the fact that $\Re w \leq 0$ if and only if $\left|\frac{w+1}{w-1}\right|<1$, we need to show that

$$
\left|\frac{1+z \frac{\left(\tilde{D}_{\alpha, \lambda}^{n} f(z)\right)^{\prime \prime}(z)}{\left(\tilde{D}_{\alpha, \lambda}^{n} f(z)\right)^{\prime}(z)}+1}{1+z \frac{\left(\tilde{D}_{\alpha, \lambda}^{n} f(z)\right)^{\prime \prime}(z)}{\left(\tilde{D}_{\alpha, \lambda}^{n} f(z)\right)(z)}-1}\right|<1 .
$$

Following the steps of proof Theorem 1, the result is straightforward.

The following two sections are concerting on the class $C_{\Sigma}(\lambda, \alpha, \gamma)$.

\section{Distortion Theorem}

The forgoing theorem obtain the the bound of $|f(z)|$ for the class $C_{\Sigma}(\lambda, \alpha, \gamma)$.

Theorem 4. Let $f(z)$ be of the form (1.4)and in the class $C_{\Sigma}(\lambda, \alpha, \gamma)$. Then for $z \in \mathbb{U}^{*}$

$$
|f(z)| \leq \frac{1}{|z|}+\sum_{k=2}^{\infty} \frac{1}{4[1+\lambda]^{n}[\gamma+(1-\gamma) C(\alpha, 2)]}|z|^{2}
$$

and

$$
|f(z)| \geq \frac{1}{|z|}+\sum_{k=2}^{\infty} \frac{1}{4[1+\lambda]^{n}[\gamma+(1-\gamma) C(\alpha, 2)]}|z|^{2} .
$$

Proof. Using Theorem 2 we have,

$4[1+\lambda]^{n}[\gamma+(1-\gamma) C(\alpha, 2)] \sum_{k=2}^{\infty}\left|a_{k}\right| \leq \sum_{k=2}^{\infty} k^{2}[1+\lambda(k-1)]^{n}[\gamma+(1-\gamma) C(\alpha, k)]\left|a_{k}\right| \leq 1$.

That is,

$$
\sum_{k=2}^{\infty}\left|a_{k}\right| \leq \frac{1}{4[1+\lambda]^{n}[\gamma+(1-\gamma) C(\alpha, 2)]}
$$




$$
\begin{aligned}
|f(z)| & =\left|\frac{1}{z}+\sum_{k=2}^{\infty} a_{k} z^{k}\right| \\
& \leq\left|\frac{1}{z}\right|+\sum_{k=2}^{\infty}\left|a_{k}\right||z|^{k} \\
& \leq \frac{1}{|z|}+\sum_{k=2}^{\infty}\left|a_{k}\right||z|^{2} \\
& \leq \frac{1}{|z|}+\sum_{k=2}^{\infty} \frac{1}{4[1+\lambda]^{n}[\gamma+(1-\gamma) C(\alpha, 2)]}|z|^{2} .
\end{aligned}
$$

The other assertion can be proved as follows

$$
\begin{aligned}
|f(z)| & =\left|\frac{1}{z}+\sum_{k=2}^{\infty} a_{k} z^{k}\right| \\
& \geq\left|\frac{1}{z}\right|+\sum_{k=2}^{\infty}\left|a_{k}\right||z|^{k} \\
& \geq \frac{1}{|z|}+\sum_{k=2}^{\infty}\left|a_{k}\right||z|^{2} \\
& \geq \frac{1}{|z|}+\sum_{k=2}^{\infty} \frac{1}{4[1+\lambda]^{n}[\gamma+(1-\gamma) C(\alpha, 2)]}|z|^{2} .
\end{aligned}
$$

This completes the proof.

\subsection{Extreme Points}

In this subsection, extreme points of the normalised concave meromorphic functions of the form (1.4) are obtained.

Theorem 5. Let $f_{1}(z)=\frac{1}{z}$ and $f_{k}(z)=\frac{1}{z}+\frac{1}{k^{2}[1+\lambda(k-1)]^{n}[\gamma+(1-\gamma) C(\alpha, k)]} z^{k}$. Then $f(z)$ concave meromorphic function of the form (1.4) if and only if it can be expressed in the form

$$
f(z)=\sum_{k=1}^{\infty} \delta_{k} f_{k}(z),
$$

where $\delta_{k} \geq 0$ and $\sum_{k=1}^{\infty} \delta_{k}=1$.

Proof. Assume that

$$
f(z)=\sum_{k=1}^{\infty} \delta_{k} f_{k}(z) .
$$


Then

$$
\begin{aligned}
f(z) & =\sum_{k=1}^{\infty} \delta_{k} f_{k}(z) \\
& =\delta_{1} \frac{1}{z}+\sum_{k=2}^{\infty} \delta_{k}\left(\frac{1}{z}+\frac{1}{k^{2}[1+\lambda(k-1)]^{n}[\gamma+(1-\gamma) C(\alpha, k)]} z^{k}\right) \\
& =\left(\sum_{k=1}^{\infty} \delta_{k}\right) \frac{1}{z}+\sum_{k=2}^{\infty} \delta_{k} \frac{1}{k^{2}[1+\lambda(k-1)]^{n}[\gamma+(1-\gamma) C(\alpha, k)]} z^{k} \\
& =\frac{1}{z}+\sum_{k=2}^{\infty} \delta_{k} \frac{1}{k^{2}[1+\lambda(k-1)]^{n}[\gamma+(1-\gamma) C(\alpha, k)]} z^{k} .
\end{aligned}
$$

Thus,

$$
\begin{gathered}
\sum_{k=2}^{\infty} \delta_{k} \frac{1}{k^{2}} k^{2} \\
=\sum_{k=2}^{\infty} \delta_{k}=1-\delta_{1}<1 .
\end{gathered}
$$

Therefore, $f(z)$ is a concave meromorphic function of the form (1.4).

Conversely, suppose that $f(z)$ is concave meromorphic function of the form (1.4). So

We can set

$$
a_{k} \leq \frac{1}{k^{2}}, \quad(k=2,3, \ldots) .
$$

$$
\begin{aligned}
& \delta_{k}:=k^{2} \\
& \delta_{1}:=1-\sum_{k=2}^{\infty} \delta_{k}=1 .
\end{aligned}
$$

Then,

$$
\begin{aligned}
f(z) & =\frac{1}{z}+\sum_{k=2}^{\infty} a_{k} z^{k} \\
& =\delta_{1} f_{1}(z)+\sum_{k=2}^{\infty} \delta_{k} f_{k}(z) \\
& =\sum_{k=1}^{\infty} \delta_{k} f_{k}(z) .
\end{aligned}
$$

This completes the proof. 
M. Al-Kaseasbeh, M. Darus - Concave Meromorphic Functions ...

Corollary 6. The extreme points of concave meromorphic functions $f(z)$ of the form (1.4) are given by $f_{1}(z)=\frac{1}{z}$ and $f_{k}(z)=\frac{1}{z}+\frac{z^{k}}{k^{2}[1+\lambda(k-1)]^{n}[\gamma+(1-\gamma) C(\alpha, k)]}$, $(k=1,2,3, \ldots)$.

Proof. The proof follows by condition (2.2).

Acknowledgement: The work here is supported by MOHE grant: FRGS/1/2016/STG06/UKM/01/1.

\section{REFERENCES}

[1] Al-Kaseasbeh, M. and Darus, M., Meromorphic functions involved constructed differential operator, Jnanabha - Vijnana Parishad of India, 47(1)(2017), 63-76.

[2] Al-Kaseasbeh, M., and Darus, M., On an operator defined by the combination of both generalized operators of Salagean and Ruscheweyh, Far East Journal of Mathematical Sciences, 97(4)(2015), 443-455.

[3] Al-Kaseasbeh, M. and Darus, M., On Concave Meromorphic Mappings, Journal of Advanced Mathematics and Applications, 6 (2017), 1-5. (in press).

[4] Al-Oboudi, F.M., On univalent functions defined by a generalized Salagean operator, International Journal of Mathematics and Mathematical Sciences, 2004(27)(2004), 1429-1436.

[5] Aouf, M. and Silverman, H., Generalizations of Hadamard products of certain meromorphic univalent functions with positive coefficients, Demonstratio Mathematica, Warsaw Technical University Institute of Mathematics, 41(2)(2008), 381-388.

[6] Bhowmik, B., Ponnusamy, S., and Wirths, K. J., Characterization and the preSchwarzian norm estimate for concave univalent functions, Monatshefte fur Mathematik, 161(1) (2010), 59-75.

[7] Chuaqui, M., Duren, P., and Osgood, B., Concave conformal mappings and prevertices of Schwarz-Christoffel mappings, Proceedings of the American Mathematical Society, 140(10)(2012), 3495-3505.

[8] Challab, K., and Darus, M., On certain class of meromorphic harmonic concave functions defined by Salagean operator, Journal of Quality Measurement and Analysis(JQMA), 11(1)(2015), 49-60.

[9] Challab, K., and Darus, M., On certain classes of meromorphic harmonic concave functions defined by Al-Oboudi operator, Journal of Quality Measurement and Analysis (JQMA), 12(1-2)(2016), 53-65.

[10] Darus, M. and K. Al-Shaqsi., Differential sandwich theorems with generalised derivative operator, International Journal of Computational and Mathematical Sciences, 2(2)(2008), 75-78. 
M. Al-Kaseasbeh, M. Darus - Concave Meromorphic Functions ...

[11] Pfaltzgraff, J. A., and Pinchuk, B., A variational method for classes of meromorphic functions, Journal d'Analyse Mathematique, 24(1)(1971), 101-150.

Mohammad Al-Kaseasbeh

School of Mathematical Sciences, Faculty of Science and Technology, Universiti Kebangsaan Malaysia 43600 UKM Bangi, Selangor, Malaysia email: zakariya.alkaseasbeh@gmail.com

Maslina Darus

School of Mathematical Sciences, Faculty of Science and Technology, Universiti Kebangsaan Malaysia 43600 UKM Bangi, Selangor, Malaysia email: maslina@ukm.edu.my (corresponding author) 